\title{
Prevalence of swallowing and speech problems in daily life after chemoradiation for head and neck cancer based on cut-off scores of the patient-reported outcome measures SWAL-QOL and SHI
}

\author{
Rico N. Rinkel ${ }^{1}$ - Irma M. Verdonck-de Leeuw ${ }^{1}$ Patricia Doornaert ${ }^{2}$. \\ Jan Buter ${ }^{3} \cdot$ Remco de Bree $^{1} \cdot$ Johannes A. Langendijk $^{4} \cdot$ Neil K. Aaronson ${ }^{5}$. \\ C. René Leemans ${ }^{1}$
}

Received: 21 August 2014/ Accepted: 31 May 2015/Published online: 14 June 2015

(C) The Author(s) 2015. This article is published with open access at Springerlink.com

\begin{abstract}
The objective of this study is to assess swallowing and speech outcome after chemoradiation therapy for head and neck cancer, based on the patient-reported outcome measures Swallowing Quality of Life Questionnaire (SWAL-QOL) and Speech Handicap Index (SHI), both provided with cut-off scores. This is a cross-sectional study. Department of Otolaryngology/Head and Neck Surgery of a University Medical Center. Sixty patients, 6 months to 5 years after chemoradiation for head and neck squamous cell carcinoma. Swallowing Quality of Life Questionnaire (SWAL-QOL) and SHI, both validated in Dutch and provided with cut-off scores. Associations were tested between the outcome measures and independent variables (age, gender, tumor stage and site, and radiotherapy technique, time since treatment, comorbidity and food intake). Fifty-two patients returned the SWAL-QOL and 47 the SHI (response rate 87 and $78 \%$, respectively). Swallowing and speech problems were present in 79 and $55 \%$, respectively. Normal food intake was noticed in 45 , $35 \%$ had a soft diet and $20 \%$ tube feeding. Patients with
\end{abstract}

Irma M. Verdonck-de Leeuw

im.verdonck@vumc.nl

1 Department of Otolaryngology/Head and Neck Surgery, VU University Medical Center, Amsterdam, The Netherlands

2 Department of Radiation Oncology, VU University Medical Center, Amsterdam, The Netherlands

3 Department of Medical Oncology, VU University Medical Center, Amsterdam, The Netherlands

4 Department of Radiation Oncology, University Medical Center Groningen/University of Groningen, Groningen, The Netherlands

5 Division of Psychosocial Research and Epidemiology, The Netherlands Cancer Institute, Amsterdam, The Netherlands soft diet and tube feeding reported more swallowing problems compared to patients with normal oral intake. Tumor subsite was significantly associated with swallowing outcome (less problems in larynx/hypopharynx compared to oral/oropharynx). Radiation technique was significantly associated with psychosocial speech problems (less problems in patients treated with IMRT). Swallowing and (to a lesser extent) speech problems in daily life are frequently present after chemoradiation therapy for head and neck cancer. Future prospective studies will give more insight into the course of speech and swallowing problems after chemoradiation and into efficacy of new radiation techniques and swallowing and speech rehabilitation programs.

Keywords Swallowing - Speech · Patient-reported outcomes - Quality of life - Head and neck cancer . Chemoradiation

\section{Introduction}

Advanced head and neck cancer is increasingly being treated with organ-preservation protocols such as chemoradiation therapy (CHRT). Organ-preservation protocols aim, next to the foremost goal to cure the disease, also at maintenance of respiration, deglutition, speech, phonation and cosmetics. However, literature reviews revealed that organ-preservation protocols often result in swallowing impairment; also speech problems may occur but these are investigated less often [1-5]. Most of the studies included in these reviews focused on swallowing impairment using videofluoroscopy, fiberoptic endoscopic evaluation of swallowing (FEES ${ }^{\mathrm{TM}}$ ), or toxicity grading protocols, but recent studies involve patient-reported 
outcomes measures as well [6-10]. Information from objective imaging techniques regarding swallowing impairment is important but does not necessarily relate to patient-reported swallowing outcomes [11-14]. There is broad range of variety of questionnaires used to evaluate swallowing and speech outcomes and the impact on quality of life [2, 15]. The head and neck cancer modules accompanying the EORTC and FACT Quality of Life Questionnaires or the University of Washington Quality of Life Questionnaire are often used. Studies using specific swallowing and speech-specific questionnaires like the MD Anderson Dysphagia Index (MDADI) [16] or the Swallowing Questionnaire on Quality of Life (SWAL-QOL) [17, 18], or the Speech Handicap index (SHI) [19] to assess speech or swallowing problems in daily life after chemoradiation therapy are less often reported.

The goal of the present cross-sectional study was to assess the prevalence of patient-reported speech and swallowing outcome after chemoradiation therapy for head and neck cancer. Measures were chosen that are provided with clear cut-off values: the Dutch versions of the Swallowing Quality of Life Questionnaire (SWALQOL) and the Speech Handicap Index (SHI), which enables quantification of patient-reported speech and swallowing problems in daily life [18, 19]. Furthermore, insight will be obtained regarding the association of sociodemographic (age and gender) and clinical factors \{comorbidity [Adult Comorbidity Evaluation 27 (ACE27)] [20], tumor site and stage, radiotherapy scheduling, time since treatment $\}$ and food intake (normal, soft diet, or tube feeding) with patient-reported speech and swallowing outcome.

\section{Materials and methods}

\section{Ethical considerations}

The study was conducted according to regular procedures of the local ethical committee of the VU University Medical Center, Amsterdam. All patients provided informed consent.

\section{Patients}

Inclusion criteria comprised primary head and neck carcinoma (all subsites, all stages) and chemoradiation treatment. One hundred and three patients with primary head and neck carcinoma underwent CHRT of which 71 were alive at the time of the study. Seven patients were excluded because of distant metastasis or loco-regional recurrence and 4 patients were excluded because they did not speak Dutch, leaving a study cohort of 60 patients.
Data on age, gender, comorbidity, and tumor and treatment characteristics [site and stage (according to UICC)], radiation technique, time since treatment, placement and removal of gastrostomy tube were collected from the medical records. Comorbidity was assessed with the Adult Comorbidity Evaluation 27 (ACE-27) [20]. The ACE-27 includes 27 comorbid conditions, including cardiovascular, respiratory, gastro-intestinal, renal, endocrine, neurological, immunological, psychiatric and rheumatologic disorders, previous or synchronous malignancy, alcohol abuse and excessive body weight. The ACE-27 was designed specifically for cancer patients and classifies patients into 4 grades of comorbidity [none (grade 0), mild (grade 1), moderate (grade 2), severe (grade 3)].

During a time span of 8 months, 52 out of 60 patients returned the SWAL-QOL (response rate $87 \%$ ) and 47, the SHI (response rate $78 \%$ ). An overview of patient characteristics is provided in Table 1 . Median age of the patients was 58 years (range 36-75). Thirty-five $(67 \%)$ of the patients were male. No comorbidity was observed in 16 patients, 24 patients had grade 1,8 patients grade 2 and 4 patients grade 3 . Primary tumor locations were oral cavity $(n=5)$, oropharynx $(n=30)$, nasopharynx $(n=4)$, larynx $(n=10)$ and hypopharynx $(n=3)$ and these were categorized into oral cavity/oropharynx/nasopharynx $(n=39)$ and larynx/hypopharynx $(n=13)$ for comparison and statistical analyses. Overall tumor stages were II $(n=2)$, III $(n=18)$ and IV $(n=32)$, which were categorized into stage II-III $(n=20)$ vs. IV $(n=32)$ for comparison and statistical analyses. Almost all (97 \%)
Table 1 Overview of patient characteristics $(n=52)$

\begin{tabular}{lc}
\hline Gender & \\
Male & $35(67 \%)$ \\
Female & $17(33 \%)$ \\
Comorbidity & \\
None & $16(31 \%)$ \\
Grade 1 & $24(46 \%)$ \\
Grade 2 & $8(15 \%)$ \\
Grade 3 & $4(8 \%)$ \\
Tumor site & \\
Oral cavity & $5(9 \%)$ \\
Oropharynx & $30(58 \%)$ \\
Nasopharynx & $4(8 \%)$ \\
Larynx & $10(19 \%)$ \\
Hypopharynx & $3(6 \%)$ \\
Tumor stage & \\
II/III & $23(44 \%)$ \\
IV & $29(56 \%)$ \\
Radiotherapy & \\
3D-CRT & $31(60 \%)$ \\
IMRT & $21(40 \%)$ \\
\hline
\end{tabular}


patients underwent PEG placement at start of treatment. All patients underwent chemoradiation, in $79 \%$ of the patients consisting of Cisplatin $100 \mathrm{mg} / \mathrm{m} 2$ in 3 cycles. Of the remaining patients, $4 \%$ received 1 cycle and $12 \%$ received 2 cycles. Three patients $(6 \%)$ underwent intraarterial Cisplatin $150 \mathrm{mg} / \mathrm{m} 2$ in 4 cycles. Chemotherapy was given concomitantly with radiotherapy ( $2 \mathrm{~Gy}$ per fraction, 5 times per week, total dose of $70 \mathrm{~Gy}$ ). One patient received also 5-Fluor-Uracil because of cisplatin side-effects after 1 cycle. In 31 patients, conventional three-dimensional conformal radiotherapy (3D-CRT) was given and intensity-modulated radiation therapy (IMRT) in 21 patients. Patients treated before 2005 were treated with 3D-CRT, without sparing the salivary glands. In 2005, IMRT was introduced as part of standard care at the department of radiation oncology enabling a significant reduction of the dose to the salivary glands. The gastrostomy tube was removed in $80 \%$ after complete remission and when oral intake was sufficient (in average 3-6 months after end of treatment). Time since treatment ranged from 6 to 58 months with a median of 21 months.

\section{Patient-reported outcomes}

Swallowing and speech impairment was measured via the Swallowing Questionnaire on Quality of Life (SWALQOL) and the Speech Handicap Index (SHI).

The SWAL-QOL is a 44-item questionnaire on swallowing-related problems in daily life [17, 18]. Response categories range on a 5-point scale. There are 10 subscales: (1) food selection ( 2 items); (2) eating duration (2 items); (3) eating desire (3 items); (4) Fear (4 items); (5) burden (2 items); (6) mental health (5 items); (7) social functioning (5 items); (8) communication (2 items); (9) sleep (2 items); and (10) fatigue (3 items). Furthermore, there is an overall symptom scale (14 items). Finally, a total SWAL-QOL Score can be calculated (based on the 23 items of the first 7 scales listed above). All SWAL-QOL scales range from 0 to 100 , a higher score indicating more impairment. Three single questions are included regarding nutrition intake ("normal", "soft", "pureed", "liquids only", "mostly tube feeding" and "tube feeding only"), liquid intake ("all liquids", "thick liquids", "very thick liquids", "thickened liquids" and "no liquids"), and general health ("poor", "moderate", "good", "very good" and "excellent"). The SWAL-QOL was translated and validated for use in Dutch head and neck cancer patients. A cut-off score on the total SWAL-QOL score of 14 points (or higher) (94\% sensitivity and $84 \%$ specificity) indicates swallowing problems in daily life [18].

The SHI is a validated speech-specific quality of life questionnaire and consists of 30-items focusing on speechrelated problems in daily life. Response categories range on a 5-point Likert scale ("never", "almost never", "sometimes", "almost always" and "always"). The questionnaire also includes an overall speech quality item, with 4 response categories ("good", "reasonable", "poor" and "severe"). A total SHI score is calculated by summing the scores on all 30 items (score range 0-120), with a higher score indicating a higher level of speech-related problems. Two subscales are distinguished: psychosocial function and speech function. A total SHI score $\geq 6$ indicates speech problems in daily life $(95 \%$ sensitivity and $90 \%$ specificity) [19].

\section{Statistical analyses}

Descriptive statistics were calculated for the SWAL-QOL and SHI scales. A $t$ test for independent groups was used to test differences between patients regarding gender (male vs. female), tumor site (larynx/hypopharynx vs. oral cavity/ oropharynx/nasopharynx), tumor stage (stage I-II vs. stage III-IV) and type of radiotherapy (3D vs. IMRT) on SWALQOL and SHI scores. Analysis of variance (ANOVA) was carried out to test differences regarding comorbidity (grade $0,1,2$ or 3) and food intake (normal, soft diet, pureed diet, tube feeding). In case of overall significant differences, post hoc analyses were carried out to pairwise compare all different groups. Bonferroni correction was applied to correct for multiple testing. Correlation analyses were performed to study the relation between age (Pearson) and time since treatment (Spearman's rank) and SWAL-QOL and SHI scores, and to study the relation between the total SWAL-QOL scale and the total SHI scale. Those variables that showed univariate significant relations with the outcome measures, were included into the multivariate regression analysis (stepwise) to obtain insight into which sociodemographic (age, gender) and clinical (comorbidity, tumor site and stage, radiation technique, time since treatment) parameters are associated with patient-reported speech and swallowing outcome. For all statistical tests, significance was defined as $p<0.05$. All analyses were performed using the IBM Statistical Package for the Social Science (SPSS) version 20 (IBM Corp., Armonk, NY USA).

\section{Results}

\section{Patient-reported swallowing outcome}

A deviant SWAL-QOL score (total SWAL-QOL score $\geq 14)$ was observed in $79 \%$ of the patients $(41 / 52)$. Mean scores are presented in Table 2. Relatively high scores were observed on the subscales general burden, food selection, eating duration, eating desire, fatigue and sleep. 
Table 2 Summary of mean scores (SD) of all patients on the subscales of the SWAL-QOL and the SHI, and regarding food intake (normal diet vs. soft/pureed vs. tube feeding), and $p$ values regarding group differences

\begin{tabular}{|c|c|c|c|c|c|c|c|c|}
\hline & \multirow{2}{*}{$\begin{array}{l}\text { All patients } \\
(n=52) \\
\text { Mean (SD) }\end{array}$} & \multirow{2}{*}{$\begin{array}{l}\text { Normal } \\
(n=23) \\
\text { Mean (SD) }\end{array}$} & \multirow{2}{*}{$\begin{array}{l}\text { Soft or pureed } \\
(n=18) \\
\text { Mean (SD) }\end{array}$} & \multirow{2}{*}{$\begin{array}{l}\text { Tube feeding } \\
(n=10) \\
\text { Mean (SD) }\end{array}$} & \multicolumn{4}{|l|}{$p$ value } \\
\hline & & & & & Overall & $\begin{array}{l}\text { Normal vs. } \\
\text { soft/pureed* }\end{array}$ & $\begin{array}{l}\text { Normal vs. } \\
\text { tube feeding* }\end{array}$ & $\begin{array}{l}\text { Soft/pureed vs. } \\
\text { tube feeding* }\end{array}$ \\
\hline $\begin{array}{l}\text { SWAL-QoL (total } \\
\text { score) }\end{array}$ & $35.8(23.8)$ & $20.0(19.7)$ & $50.6(17.9)$ & $48.5(17.3)$ & $<0.001$ & $<0.001$ & 0.001 & 1.00 \\
\hline $\begin{array}{l}\text { Symptomen } \\
\text { schaal van de } \\
\text { SHI }\end{array}$ & $35.4(19.5)$ & $23.8(19.0)$ & $43.7(15.3)$ & $49.6(10.7)$ & $<0.001$ & 0.001 & 0.001 & 1.00 \\
\hline General burden & $42.7(30.4)$ & $25.1(29.2)$ & $60.6(22.8)$ & $52.6(24.2)$ & $<0.001$ & $<0.001$ & 0.023 & 1.00 \\
\hline Food selection & $45.8(34.0)$ & $20.7(24.6)$ & $69.0(20.1)$ & $66.5(30.0)$ & $<0.001$ & $<0.001$ & $<0.001$ & 1.00 \\
\hline Eating duration & $56.9(32.2)$ & $38.2(30.1)$ & 73.7 (21.0) & $72.6(31.5)$ & $<0.001$ & $<0.001$ & 0.005 & 1.00 \\
\hline Eating desire & 39.8 (31.9) & $19.5(27.5)$ & $51.3(20.6)$ & $69.9(25.0)$ & $<0.001$ & 0.001 & $<0.001$ & 0.19 \\
\hline Fear of eating & $30.2(24.4)$ & $21.8(22.0)$ & $42.6(22.2)$ & $30.1(25.8)$ & 0.021 & 0.017 & 1.00 & 0.52 \\
\hline Sleep & $49.5(31.3)$ & $39.8(29.7)$ & $59.3(25.6)$ & $53.9(41.3)$ & 0.13 & 0.15 & 0.71 & 1.00 \\
\hline Fatigue & $47.1(24.3)$ & $43.1(29.2)$ & 47.7 (17.2) & $55.0(24.3)$ & 0.44 & 1.00 & 0.62 & 1.00 \\
\hline Communication & $32.3(29.2)$ & $20.7(23.8)$ & $38.3(30.4)$ & $46.3(32.4)$ & 0.034 & 0.15 & 0.059 & 1.00 \\
\hline Mental health & $30.6(26.5)$ & $16.1(19.8)$ & $44.4(22.2)$ & $41.0(31.6)$ & 0.001 & 0.001 & 0.021 & 1.00 \\
\hline Social FX & $28.8(25.8)$ & $13.5(17.1)$ & $43.3(26.0)$ & $40.5(22.5)$ & $<0.001$ & $<0.001$ & 0.006 & 1.00 \\
\hline SHI (total score) & $18.6(21.8)$ & $11.7(15.2)$ & $22.8(27.7)$ & $29.3(22.6)$ & 0.11 & 0.38 & 0.19 & 1.00 \\
\hline $\begin{array}{l}\text { SHI psychosocial } \\
\text { subscale }\end{array}$ & $6.7(10.7)$ & $3.0(6.4)$ & $10.2(13.7)$ & $10.3(11.2)$ & 0.069 & 0.11 & 0.33 & 1.00 \\
\hline $\begin{array}{l}\text { SHI speech } \\
\text { quality subscale }\end{array}$ & $11.8(12.0)$ & $8.4(10.1)$ & $12.7(13.5)$ & 18.7 (11.8) & 0.12 & 0.84 & 0.14 & 0.79 \\
\hline
\end{tabular}

Statistical significant differences $(p<0.05)$ are printed bold

* $p$ values are corrected with the Bonferroni correction to account for multiple testing

Normal food intake was reported by 23 patients $(45 \%)$, while $18(35 \%)$ took a soft diet or pureed food, and 10 (20\%) had tube feeding (data were missing for 1 patient). Patients with normal food intake had significantly better mean scores compared to patients with soft diet or tube feeding on all SWAL-QoL scales, except for the subscales sleep and fatigue; patients with soft diet or tube feeding did not differ from each other significantly on any of the scales (Table 2).

Univariate analyses revealed that age, gender, comorbidity and tumor stage were not significantly related to SWAL-QOL scores. Swallowing outcome was significantly associated with tumor site and radiotherapy technique. Patients treated for a laryngeal or hypopharyngeal tumor had significantly $(p<0.05)$ better scores compared to patients treated for an oral cavity, oropharyngeal or nasopharynx tumor on the total SWAL-QOL $(d f=50$; $t=-2.10 ; p=0.041)$ and the subscales general burden $(d f=50 ; t=-2.23 ; p=0.030)$, mental health $(d f=50$; $t=-2.30 ; p=0.026)$ and social functioning $(d f=50$; $t=-2.46 ; p=0.017)$. Compared to 3D-CRT, patients after IMRT had significantly better scores on the total SWAL-QOL $\quad(d f=49.9 ; \quad \mathrm{t}=2.22 ; \quad p=0.031), \quad$ food selection $(d f=50 ; t=2.01 ; p=0.05)$, fear of eating $(d f=50 ; t=3.34 ; p=0.002)$, sleep $(d f=50 ; t=2.89$; $p=0.006)$ and social functioning $(d f=50 ; t=2.46$; $p=0.018$ ). Furthermore, a positive correlation (more problems on the long term) was found between time since treatment and the following SWAL-QOL subscales:fear of eating ( $\rho=0.57 ; p<0.001)$, sleep $(\rho=0.41 ; p=0.002)$, fatigue $(\rho=0.33 ; p=0.015)$, social function $(\rho=0.33$; $p=0.019)$ and the total SWAL-QOL score $(\rho=0.37$; $p=0.007)$.Because radiotherapy technique was related to time since treatment, correlation coefficients were also calculated in these subgroups (3D-CRT vs. IMRT) and no significant relations between time since treatment and swallowing outcome were found.

For the multivariable regression analysis only tumor site and radiotherapy technique were included in the selection procedure, which revealed that only tumor site was significantly associated with the total SWAL-QOL score $\left(B=15.5, R^{2}=0.081, F=4.40, p=0.041\right)$. Note that radiotherapy technique is univariate more significantly associated to total SWAL-QOL, but this factor did not enter the multivariate model due to unequal variances of the two groups. 


\section{Patient-reported speech outcome}

A deviant SHI score (total SHI score $\geq 6$ ) was observed in $55 \%$ of the patients (26/47). Mean scores are presented in Table 2. Univariate analyses revealed that radiotherapy technique was associated significantly with the subscale SHI psychosocial function: patients treated with IMRT had a better score $(d f=39.7 ; t=2.48 ; p=0.017)$. Age, gender, comorbidity, tumor stage and site, and time since treatment (corrected for radiotherapy technique) were not significantly associated with SHI total scores. Therefore, no multivariate regression analyses were carried out.

\section{Relation between swallowing and speech outcome}

A significant relation between swallowing and speech outcome was found: Pearson $\mathrm{r}$ was $0.56, p<0.001$ regarding the total scores on the SWAL-QOL and SHI. Regarding the presence of swallowing and speech problems (score above cut-off value), $51 \%$ of the patients after chemoradiation had both speech and swallowing problems, $24 \%$ had swallowing problems but no speech problems, $7 \%$ had speech problems but no swallowing problems and $18 \%$ had no speech or swallowing problems.

\section{Discussion}

The present study revealed a high prevalence of patientreported swallowing $(79 \%)$ and speech $(55 \%)$ problems after chemoradiation for advanced head and neck cancer. Swallowing and speech problems were significantly related to each other, indicating that many patients who experience swallowing problems also experience speech problems. The prevalence of swallowing and speech problems after (chemo)radiotherapy in earlier studies differ considerably depending among others on the assessment methods [2, 15]. Unlike earlier studies, the percentages in the present study are based on validated cut-off values of the SWALQOL and SHI questionnaires.

Swallowing problems can lead to clinically apparent as well as silent aspiration or continued alternate feeding such as feeding tube placement [33,34]. Recently, Kano et al. [35] compared the need for tube feeding support among patients treated with surgery $(26 \%)$ vs. chemoradiation (12\%), as assessed immediately after initial treatment. In the present study, $20 \%$ of the patients after CHRT had tube feeding, almost all within 18 months after treatment, which falls in the broad range as reported in the literature varying from 20 to $60 \%$ use of a feeding tube at 1 year after treatment to $8-18 \%$ longer term use [36, 37]. As expected, patients with tube feeding as well as patients with soft or pureed diet in the present study had more swallowing problems compared to patients with normal oral food intake. Dysphagia is known to have a major impact on quality of life, in particular in patients with tube feeding [38-40]. Severe dysphagia is also related to increased emotional distress, not only of the patients themselves but also of their spouses [41, 42].

In the present study, swallowing and speech problems were significantly related to tumor subsite (patients treated for oral or oropharynx cancer had significantly more swallowing problems compared to patients treated for larynx or hypopharynx cancer) and radiation technique (patients treated with IMRT reported less swallowing and speech problems). There are several causes that may explain these differences. Detailed studies on swallowing after (chemo)-radiation for head and neck cancer revealed a large variety of motility disorders, including prolonged oral transit time, decreased tongue strength/control, reduced base of tongue contact to the pharyngeal wall, pharyngeal constrictor dysmotility, decreased laryngeal elevation, reduced hyoid movement and epiglottic dysmotility [2125]. The probability of swallowing dysfunction after (chemo)radiation appears to be associated with radiationinduced thickening of the pharyngeal constrictor muscles, the supraglottic larynx and the glottic larynx [26, 27]. Furthermore a clear relationship is found between dose distributions in the salivary glands and subjective xerostomia $[28,29]$. Chemotherapy functions as a sensitizer for radiotherapy and enhances the effects of radiotherapy on the tumor and the surrounding tissue. The cytotoxic effects of chemotherapy alone on oral, pharyngeal and laryngeal mucosa also lead to oral mucositis, infections, xerostomia, and neutropenia are associated with long-term swallowing problems, not only in head and neck cancer patients [30, $31]$, but also in, for example, breast, colon or lung cancer patients [32].

The prevalence of swallowing dysfunction and the major impact of this side effect on the more general dimensions of health-related quality of life stresses the importance of effective preventive measures and/or therapeutic interventions. New radiation delivery techniques aiming at sparing of anatomical structures that are correlated with swallowing may contribute to prevent long-term radiation-induced dysphagia [43-45]. Another possibility is speech and swallowing rehabilitation. In usual care, rehabilitation includes pretreatment evaluation of swallowing and counseling allowing to determine the swallowing status at start and to prepare the patient regarding possible swallowing impairment and the rehabilitation process and post treatment speech and swallowing management strategies consisting of oromotor exercises (to increase the strength and mobility of the lips, tongue and mandible), swallow maneuvers (to facilitate swallowing function and to prevent aspiration) and compensation techniques 
(adjusting posture, adjusting food consistency). It has been argued that patients should be encouraged to swallow throughout their treatment also when prophylactic feeding tube is placed. Recent studies reveal that exercises in an early stage, before and during radiotherapy, may prevent or decrease swallowing dysfunction after curative (chemo)radiation [5, 46-49], but not all studies show these beneficial effects [50]. There is growing evidence that attention is needed for the individual needs of the patients to determine the best rehabilitation strategy [51, 52]. Prospective randomized trials are needed to provide evidence-based effectiveness of these approaches.

It can be concluded that swallowing and (to a lesser extent) speech problems in daily life are frequently reported by patients after chemoradiation therapy for advanced head and neck cancer. Future prospective studies will give more insight into the course of speech and swallowing problems after chemoradiation and into efficacy of new radiation techniques and swallowing and speech rehabilitation.

Conflict of interest The authors declare that do not have any conflict of interest.

Open Access This article is distributed under the terms of the Creative Commons Attribution 4.0 International License (http://creativecommons.org/licenses/by/4.0/), which permits unrestricted use, distribution, and reproduction in any medium, provided you give appropriate credit to the original author(s) and the source, provide a link to the Creative Commons license, and indicate if changes were made.

\section{References}

1. Wall LR, Ward EC, Cartmill B, Hill AJ (2013) Physiological changes to the swallowing mechanism following (chemo)radiotherapy for head and neck cancer: a systematic review. Dysphagia 28(4):481-493

2. Dysphagia Section, Oral Care Study Group, Multinational Association of Supportive Care in Cancer (MASCC)/International Society of Oral Oncology (ISOO), Raber-Durlacher JE, Brennan MT, Verdonck-de Leeuw IM et al (2012) Swallowing dysfunction in cancer patients. Support Care Cancer 20(3): 433-443

3. Genden EM, Ferlito A, Rinaldo A et al (2008) Recent changes in the treatment of patients with advanced laryngeal cancer. Head Neck 30:103-110

4. Mouw KW, Haraf DJ, Stenson KM et al (2010) Factors associated with long-term speech and swallowing outcomes after chemoradiotherapy for locoregionally advanced head and neck cancer. Arch Otolaryngol Head Neck Surg 136:1226-1234

5. van der Molen L, van Rossum MA, Burkhead LM et al (2009) Functional outcomes and rehabilitation strategies in patients treated with chemoradiotherapy for advanced head and neck cancer: a systematic review. Eur Arch Otorhinolaryngol 266:889-900

6. Erkal EY, Canoğlu D, Kaya A et al (2014) Assessment of early and late dysphagia using videofluoroscopy and quality of life questionnaires in patients with head and neck cancer treated with radiation therapy. Radiat Oncol 9:137
7. Lazarus CL, Husaini H, Hu K et al (2014) Functional outcomes and quality of life after chemoradiotherapy: baseline and 3 and 6 months post-treatment. Dysphagia 29(3):365-375

8. Hunter KU, Lee OE, Lyden TH et al (2014) Aspiration pneumonia after chemo-intensity-modulated radiation therapy of oropharyngeal carcinoma and its clinical and dysphagia-related predictors. Head Neck 36(1):120-125

9. van der Molen L, Heemsbergen WD, de Jong R et al (2013) Dysphagia and trismus after concomitant chemo-intensity-modulated radiation therapy (chemo-IMRT) in advanced head and neck cancer; dose-effect relationships for swallowing and mastication structures. Radiother Oncol 106(3):364-369

10. Hunter KU, Schipper M, Feng FY (2013) Toxicities affecting quality of life after chemo-IMRT of oropharyngeal cancer: prospective study of patient-reported, observer-rated, and objective outcomes. Int J Radiat Oncol Biol Phys 85(4):935-940

11. Rogus-Pulia NM, Pierce M, Mittal BB et al (2014) Bolus effects on patient awareness of swallowing difficulty and swallow physiology after chemoradiation for head and neck cancer. Head Neck. doi:10.1002/hed.23720

12. Rogus-Pulia NM, Pierce MC, Mittal BB et al (2014) Changes in swallowing physiology and patient perception of swallowing function following chemoradiation for head and neck cancer. Dysphagia 29(2):223-233

13. Logemann JA, Pauloski BR, Rademaker AW et al (2008) Swallowing disorders in the first year after radiation and chemoradiation. Head Neck 30:148-158

14. Ding R, Logemann JA (2008) Patient self-perceptions of swallowing difficulties as compared to expert ratings of videofluorographic studies. Folia Phoniatr Logop 60:142-150

15. Verdonck-de Leeuw IM, van Nieuwenhuizen A, Leemans CR (2012) The value of quality-of-life questionnaires in head and neck cancer. Curr Opin Otolaryngol Head Neck Surg 20(2):142-147

16. Chen AY, Frankowski R, Bishop-Leone J et al (2001) The development and validation of a dysphagia-specific quality-oflife questionnaire for patients with head and neck cancer: the M. D. Anderson dysphagia inventory. Arch Otolaryngol Head Neck Surg 127:870-876

17. McHorney CA, Robbins J, Lomax K et al (2002) The SWALQOL and SWAL-CARE outcomes tool for oropharyngeal dysphagia in adults: III. Documentation of reliability and validity. Dysphagia 17:97-114

18. Rinkel RN, Verdonck-de Leeuw IM, van Reij EJ et al (2008) Speech Handicap Index in patients with oral and pharyngeal cancer: better understanding of patients' complaints. Head Neck 30:868-874

19. Rinkel RN, Verdonck-de Leeuw IM, Langendijk JA et al (2009) The psychometric and clinical validity of the SWAL-QOL questionnaire in evaluating swallowing problems experienced by patients with oral and oropharyngeal cancer. Oral Oncol 45:e67e71

20. Piccirillo JF (2000) Impact of comorbidity and symptoms on the prognosis of patients with oral carcinoma. Arch Otolaryngol Head Neck Surg 126:1086-1088

21. Goguen LA, Posner MR, Norris CM et al (2006) Dysphagia after sequential chemoradiation therapy for advanced head and neck cancer. Otolaryngol Head Neck Surg 134:916-922

22. Eisbruch A, Lyden T, Bradford CR et al (2002) Objective assessment of swallowing dysfunction and aspiration after radiation concurrent with chemotherapy for head-and-neck cancer. Int J Radiat Oncol Biol Phys 53:23-28

23. Kotz T, Costello R, Li Y et al (2004) Swallowing dysfunction after chemoradiation for advanced squamous cell carcinoma of the head and neck. Head Neck 26:365-372

24. Ku PK, Yuen EH, Cheung DM et al (2007) Early swallowing problems in a cohort of patients with nasopharyngeal carcinoma: 
symptomatology and video fluoroscopic findings. Laryngoscope 117:142-146

25. Hughes PJ, Scott PM, Kew J et al (2000) Dysphagia in treated nasopharyngeal cancer. Head Neck 22:393-397

26. Eisbruch A, Kim HM, Feng FY et al (2011) Chemo-IMRT of oropharyngeal cancer aiming to reduce dysphagia: swallowingorganslatecomplicationprobabilitiesanddosimetriccorrelates. Int J Radiat Oncol Biol Phys 81(3):e93-e99

27. Christianen ME, Schilstra C, Beetz I et al (2012) Predictive modelling for swallowing dysfunction after primary (chemo)radiation: results of prospective observational study. Radiother Oncol 105(1):107-114

28. Beetz I, Schilstra C, Visink A et al (2013) Role of minor salivary glands in developing patient-rated xerostomia and sticky saliva during day and night. Radiother Oncol 109(2):311-316

29. Little M, Schipper M, Feng FY et al (2012) Reducing xerostomia after chemo-IMRT for head-and-neck cancer: beyond sparing the parotid glands. Int J Radiat Oncol Biol Phys 83(3):1007-1014

30. Trotti A, Bellm LA, Epstein JB et al (2003) Mucositis incidence, severity and associated outcomes in patients with head and neck cancer receiving radiotherapy with or without chemotherapy: a systematic literature review. Radiother Oncol 66:253-262

31. Cartmill B, Cornwell P, Ward E et al (2012) Swallowing, nutrition and patient-rated functional outcomes at 6 months following two non-surgical treatments for T1-T3 oropharyngeal cancer. Support Care Cancer 20:2073-2081

32. De Ruysscher D, Dehing C, Bremer RH et al (2007) Maximal neutropenia during chemotherapy and radiotherapy is significantly associated with the development of acute radiation-induced dysphagia in lung cancer patients. Ann Oncol 18:909-916

33. Nguyen NP, Frank C, Moltz CC et al (2006) Aspiration rate following chemoradiation for head and neck cancer: an underreported occurrence. Radiother Oncol 80:302-306

34. Costa Bandeira AK, Azevedo EH, Vartanian JG et al (2008) Quality of life related to swallowing after tongue cancer treatment. Dysphagia 23:183-192

35. Kano S, Homma A, Hayashi R et al (2013) Matched-pair analysis in patients with advanced oropharyngeal cancer: surgery vs. concurrent chemoradiotherapy. Oncology 84(5):290-298

36. Myers C, Kerr P, Cooke A et al (2012) Functional outcomes after treatment of advanced oropharyngeal carcinoma with radiation or chemoradiation. J Otolaryngol Head Neck Surg 41:108-118

37. Frowen JJ, Perry AR (2006) Swallowing outcomes after radiotherapy for head and neck cancer: a systematic review. Head Neck 28:932-944

38. Langendijk JA, Doornaert P, Verdonck-de Leeuw IM et al (2008) Impact of late treatment-related toxicity on quality of life among patients with head and neck cancer treated with radiotherapy. J Clin Oncol 26:3770-3776

39. Jensen K, Bonde Jensen A, Grau C (2006) The relationship between observer-based toxicity scoring and patient assessed symptom severity after treatment for head and neck cancer. A correlative cross sectional study of the DAHANCA toxicity scoring system and the EORTC quality of life questionnaires. Radiother Oncol 78:298-305

40. Kulbersh BD, Rosenthal EL, McGrew BM et al (2006) Pretreatment, preoperative swallowing exercises may improve dysphagia quality of life. Laryngoscope 116:883-886

41. Lin BM, Starmer HM, Gourin CG (2012) The relationship between depressive symptoms, quality of life, and swallowing function in head and neck cancer patients 1 year after definitive therapy. Laryngoscope 122(7):1518-1525

42. Verdonck-de Leeuw IM, Eerenstein SE, Van der Linden MH et al (2007) Distress in spouses and patients after treatment for head and neck cancer. Laryngoscope 117:238-241

43. Paleri V, Roe JW, Strojan P et al (2014) Strategies to reduce long-term postchemoradiation dysphagia in patients with head and neck cancer: an evidence-based review. Head Neck 36(3):431-443

44. Pauloski BR, Rademaker AW, Logemann JA et al (2014) Comparison of swallowing function after intensity-modulated radiation therapy and conventional radiotherapy for head and neck cancer. Head Neck. doi:10.1002/hed.23796

45. Lohia S, Rajapurkar M, Nguyen SA et al (2014) A comparison of outcomes using intensity-modulated radiation therapy and 3-dimensional conformal radiation therapy in treatment of oropharyngeal cancer.JAMA. Otolaryngol Head Neck Surg 140(4): 331-337

46. Kraaijenga SA, van der Molen L, Jacobi I et al (2014) Prospective clinical study on long-term swallowing function and voice quality in advanced head and neck cancer patients treated with concurrent chemoradiotherapy and preventive swallowing exercises. Eur Arch Otorhinolaryngol [Epub ahead of print]

47. Ohba S, Yokoyama J, Kojima M et al (2014) Significant preservation of swallowing function in chemoradiotherapy for advanced head and neck cancer by prophylactic swallowing exercise. Head Neck. doi:10.1002/hed.23913

48. Duarte VM, Chhetri DK, Liu YF et al (2013) Swallow preservation exercises during chemoradiation therapy maintains swallow function. Otolaryngol Head Neck Surg 149(6):878-884

49. Carroll WR, Locher JL, Canon CL et al (2008) Pretreatment swallowing exercises improve swallow function after chemoradiation. Laryngoscope 118:39-43

50. Lazarus CL, Husaini H, Falciglia D et al (2014) Effects of exercise on swallowing and tongue strength in patients with oral and oropharyngeal cancer treated with primary radiotherapy with or without chemotherapy. Int $\mathrm{J}$ Oral Maxillofac Surg 43(5):523-530

51. Govender R, Breeson L, Tuomainen J et al (2013) Speech and swallowing rehabilitation following head and neck cancer: are we hearing the patient's voice? Clin Otolaryngol 38:433-437

52. Bradley PT, Bradley PJ (2012) Treatment of hypopharyngeal carcinoma with primary chemoradiotherapy: functional morbidity. Curr Opin Otolaryngol Head Neck Surg 20:89-96 